\title{
Basic and Translational Research on Proteinase-Activated Receptors: Preface
}

\author{
Katsuya Hirano $^{1, *}$ and Atsufumi Kawabata ${ }^{2}$ \\ ${ }^{I}$ Division of Molecular Cardiology, Research Institute of Angiocardiology, Graduate School of Medical Sciences, \\ Kyushu University, Fukuoka 812-8582, Japan \\ ${ }^{2}$ Division of Pharmacology and Pathophysiology, Kinki University School of Pharmacy, Higashi-Osaka 577-8502, Japan
}

Received July 27, 2008; Accepted September 2, 2008

Keywords: proteinase-activated receptor (PAR), pathophysiology, translational research

Proteinase-activated receptors (PARs) compose a unique family of $G$ protein-coupled receptors that mediate various cellular effects of proteinases. PARs are distinguished from other conventional $G$ protein-coupled receptors by their unique mechanism of activation. The activation of PARs is initiated by cleavage of the Nterminal extracellular region at a specific site by the agonist proteinases. The uncovered, new N-terminal region then acts as a tethered ligand, thereby activating the receptor. The identification of this type of receptor established the new role of proteinases as a signaling molecule, while also providing a molecular explanation for the known cellular effects of proteinases. In 1991, thrombin receptor, $\mathrm{PAR}_{1}$, was first identified in platelets. Thereafter, three more members, $\mathrm{PAR}_{2}, \mathrm{PAR}_{3}$, and $\mathrm{PAR}_{4}$, have been identified. $\mathrm{PAR}_{1}, \mathrm{PAR}_{3}$, and $\mathrm{PAR}_{4}$ are known to serve as receptors for thrombin; and $\mathrm{PAR}_{1}$, $\mathrm{PAR}_{2}$, and $\mathrm{PAR}_{4}$ are known to serve as receptors for trypsin. However, any proteinase with the ability to cleave the activation site can serve as an agonist.

PARs are expressed in wide variety of organs, tissues, and cells, including the nervous system, blood cells, immune system, cardiovascular system, airways, alimentary tract, reproductive organs, and so on. PARs thus play physiological and pathophysiological roles in the specialized function in each system. The expression pattern of the four subtypes of PARs as well as their relevant agonist proteinases may differ depending on the type of cell. For example, thrombin plays an important role in the cardiovascular system, and $\mathrm{PAR}_{1}$ is the major receptor mediating the cardiovascular effects of thrombin such as platelet activation, endothelial NO production,

*Corresponding author. khirano@molcar.med.kyushu-u.ac.jp Published online in J-STAGE

doi: $10.1254 /$ jphs.08R05FM and smooth muscle contraction. Nevertheless, the activity of proteinases is, in general, suppressed under physiological conditions, while it is activated under pathological situations such as hemorrhage, inflammation, and tissue damage. Therefore, PARs are suggested to play more important roles under pathological conditions. PARs are thus anticipated to serve as a new therapeutic target. The development of drugs that directly stimulate or antagonize PARs might be a prerequisite for the establishment of the therapeutic strategies targeting PARs. Antagonists for $\mathrm{PAR}_{2}$ and $\mathrm{PAR}_{4}$ as well as inhibitors of receptor signaling, which are based on the amino acid sequences of the intracellular 3rd loop of PARs, still remain at the experimental stage. In contrast, the development of $\mathrm{PAR}_{1}$ antagonists has already reached clinical trials. After more than a decade of basic research, PARs have now come of age. It may now be the time to translate basic knowledge into clinical applications.

The following mini-reviews are summaries from the symposium titled "Basic and Translational Research on Proteinase-Activated Receptors, Seeking Out a New Therapeutic Target", which was held at the 81st Annual Meeting of The Japanese Pharmacological Society in Yokohama, Japan, on March 17, 2008. At the symposium, five topics were selected from different fields of research, including neuroscience, gastrointestinal medicine, reproductive and gynecological medicine, neurosurgery, and cardiovascular medicine. The recent advances in each field were reviewed and the potential for the therapeutic application of PARs were discussed, using the on-going clinical trials of $\mathrm{PAR}_{1}$ antagonist as a prototype.

In this series, 1) Dr. Nagai et al. will summarize the $\mathrm{PAR}_{1}$-mediated modulation of the nicotine-induced rewarding effect in the nucleus accumbens. They will 
discuss the possibility of a novel therapeutic approach for the treatment of nicotine dependence by targeting the tissue plasminogen-plasmin-PAR ${ }_{1}$ system in the central nervous system. 2) Drs. Yoshida and Yoshikawa will summarize the roles of proteinases and PARs in the gastrointestinal inflammatory diseases. They will discuss a proinflammatory role of $\mathrm{PAR}_{2}$ in reflux esophagitis and the roles of $\mathrm{PAR}_{1}$ and $\mathrm{PAR}_{2}$ in Helicobacter pylori gastritis. They will also discuss the therapeutic effect of protease inhibitors and antagonists for PARs on intestinal mucosal inflammation, thus proposing the potential efficacy of anti-proteinases and anti-PARs strategies for the treatment of the gastrointestinal inflammatory diseases. 3) Dr. Osuga et al. will discuss the functional roles of PARs in the female reproductive organs, with a focus on the proinflammatory effects of $\mathrm{PAR}_{1}$ and $\mathrm{PAR}_{2}$ in granulosa cells of ovary and endometrial stromal cells. They will suggest not only the physiological roles of $\mathrm{PAR}_{1}$ in the ovulatory process and $\mathrm{PAR}_{2}$ in menstruation-related changes in the endometrium, but also the pathological role of $\mathrm{PAR}_{1}$ and
$\mathrm{PAR}_{2}$ in endometriosis. 4) Dr. Kai et al. will summarize the current understanding of the molecular mechanism of cerebral vasospasm in subarachnoid hemorrhage and discuss the limitations of the current therapeutic strategies for cerebral vasospasm. They will provide a novel view of the molecular mechanism of cerebral vasospasm, which involves thrombin and $\mathrm{PAR}_{1}$ as key molecules, thereby proposing the potential of a $\mathrm{PAR}_{1}$ antagonist in a therapeutic strategy for the prevention and treatment of cerebral vasospasms. 5) Dr. Chintala et al. will summarize the current status of the development of a new $\mathrm{PAR}_{1}$ antagonist and the preliminary results of the phase II clinical trial of this $\mathrm{PAR}_{1}$ antagonist as a novel anti-platelet agent.

We hope that this mini-review series will be helpful not only for obtaining a better understanding of recent advances in the basic and translational research on PARs in the fields selected for the symposium, but also by encouraging interdisciplinary collaboration and further applications of PARs as therapeutic targets in other fields of clinical science. 